\title{
A multidisciplinary approach to the management of liver disease and alcohol disorders in psychiatric settings (Review)
}

\author{
SIMONA TRIFU ${ }^{1}$, ANDRIAN ȚÎBÎRNA ${ }^{2^{*}}$, RADU-VIRGIL COSTEA ${ }^{3 *}$ and ALEXANDRA POPESCU ${ }^{2 *}$ \\ ${ }^{1}$ Department of Clinical Neurosciences, 'Carol Davila' University of Medicine and Pharmacy, 020021 Bucharest; \\ ${ }^{2}$ Department of Psychiatry, 'Alex. Obregia' Clinical Hospital for Psychiatry, 041914 Bucharest; \\ ${ }^{3}$ Department of General Surgery, 'Carol Davila' University of Medicine and Pharmacy, 020021 Bucharest, Romania
}

Received October 23, 2020; Accepted November 24, 2020

DOI: $10.3892 /$ etm.2021.9702

\begin{abstract}
Society is burdened with the uncontrolled use of alcohol, an ongoing issue, with a substantial associated morbidity and a pressing economical reverberation. It is inevitable that a series of psychiatric patients who display alcohol disorders will be admitted to hospital while also suffering from health conditions, such as liver disease, due to the consumption of alcohol. Managing comorbid patients in a psychiatric facility is a delicate matter that requires a collaborative team. The aim of this systematic paper is to highlight the following: The possibility of treating alcohol use disorder (AUD) and alcohol withdrawal syndrome (AWS) overlapping alcohol liver disease (ALD) within a psychiatric institution, and the importance of a collaborative multidisciplinary team; correctly dosing psychoactive medication when metabolism is affected by ALD; deciding when is it necessary to seek a transfer to a general hospital. Prescribing medication in patients suffering from ALD is still a not a fully documented territory. Protein binding, metabolism, bioavailability, extraction ratios, excretion route, and half-life must be taken into consideration as well as frequently repeating liver panels. Studies suggest that short-acting benzodiazepines are preferred over their alternatives when treating AWS in ALD. All anticonvulsants can be used in patients with decompensated liver disease with caution, although newer generation antiepileptic agents should be first line. Propofol is favored to benzodiazepines or opioids in the case of decompensated cirrhosis. Patients with ALD are likely to be further compromised by the potential hepatocytotoxicity of some pharmacological agents. On that account, having an integrated perspective of the medical case while taking into
\end{abstract}

Correspondence to: Dr Radu-Virgil Costea, Department of General Surgery, 'Carol Davila' University of Medicine and Pharmacy, 37 Dionisie Lupu Street, 020021 Bucharest, Romania E-mail:radu.costea@umfcd.ro

*Contributed equally

Key words: alcohol use disorder, alcohol liver disease, albumin, benzodiazepine, propofol consideration the underlying illness as well as possible drug interaction is crucial in treating AUD or AWS in a psychiatric institution.

\section{Contents}

1. Introduction

2. Medication and protocols

3. Correlation of treatment under psychiatric environment

4. Conclusion

\section{Introduction}

The cause of alcohol use disorder (AUD) is unknown. Therefore, we can only talk about assumptions, including psychological, sociological, personality structure, biochemical and genetic.

Psychological assumption (psychological factors) enjoys the highest reliability (credibility); however, it does not enjoy scientific validity. Factors such as frustration, stressful events, or losses are more easily borne by alcohol ingestion (alcohol increases self-esteem) (1).

Sociological assumption (sociological factors)-alcohol consumption was determined by habits, lifestyles, as well as the psychology of the individual: Insistence on alcohol consumption; social convention (one drinks a glass when meeting in certain groups); skill. Sociological factors include 'drinking pressure' through strong peer pressure (2).

Assumption of personality structure (personality factors)-there are certain structures, certain individualswho overcome with difficulty or cannot overcome trauma, loss, frustration, or failure. Consequently, they resort to alcohol as a kind of psychological crutch based on the personality type (hysterical, dysthymic, cyclothymic, depressive, antisocial personalities) (3).

Biochemical refers to theneurobiological theory: At the base of the brain, in the diencephalon there are certain neural structures with double function: Receptors and secretors. These formations secrete enkephalins, precursors of endorphins-with an opiate-like chemical structure-that ensure peace and balance. In some individualsthe number of receptors is too 
small, in others the receptors have poor functioning capacity, secretory capacity, insufficient to ensure peace, balance, or sedation. The exogenous intake of alcohol or drugs completes the functional capacity of the mentioned receptors, in the cerebrospinal fluid being low: GABA, serotonin, dopamine (4). Consumption owing to solitude or feelings thereof also constitutes a significant step towards addiction (5).

The so-called enzyme assumption isin close correlation with the biochemical assumption andexplains why blood alcohol levels are variable at the same amount ingested, as well as the fact that resistance to high blood alcohol levels varies. Biochemical assumption, unlike the psychological one, enjoys the greatest scientific validity; this is termed alcohol tolerance, as well as cross-tolerance (e.g., anesthetic resistance). Alcohol is an enzyme inducer in the microsomes of the liver. When consuming alcohol there is an overdevelopment of the enzyme system and the basal metabolism (BM) is higher. Alcoholics who have preserved reality testing and are aware of what is happening to them, tolerates high doses of Phenobarbital because hepatic enzymes are increased, BMis increased, and the individual becomes resistant to anesthetics. If inebriated, an additive effect occurs: Alcohol and Phenobarbital enter into hepatic competition for BM and may increase serum Phenobarbital levels to toxic and dangerous values (6).

Genetic assumption refers to alcoholic parents, which determines a 3- to 4-fold higher risk in children asthere is a greater concordance in monozygotes than in dizygotes. Thus,children of alcoholic parents retain the risk of developing an alcohol-related disorder, even if they are adopted by non-alcoholics or vice versa albeitchildren of non-alcoholics adopted by alcoholics are not at risk of alcoholism. A family history increases the risk of alcoholism, while genetics play a greater role thanthe environment (7).

According to data released by the NHS 50\% of acute psychiatric hospitalized patients are diagnosed with AUD and over $20 \%$ are alcohol-dependent (8). Recent USA specific reports published by the American Addiction Centers indicate that an average of $40 \%$ of all hospital beds are occupied by patients with alcohol-related disease. Additionally, on average within the mortality caused by liver disease $47.8 \%$ was associated with alcohol (9).

When decreasing or ceasing alcohol intake after chronic abuse of substance there is a risk of developing alcohol withdrawal syndrome (AWS) and delirium tremens (DT) (10). Withdrawal Syndrome is common but not unanimous, $50 \%$ of alcoholics will suffer from withdrawal symptoms. Approximately 5-20\% of patients withdrawing from alcohol experience Delirium Tremens (11).

In comparison to other European states, Romania is one of the largest alcohol-consuming countries with more than 12.5 liters of pure alcohol per capita (12). Yet a brief local study showed limited statistical studies pertaining to AUDs per se (13). A possible explanation is that approximately $25 \%$ of alcohol consumed is not registered, it being produced, consumed and distributed outside of governmental control, according to the WHO.

AUD is a chronic pathological pattern of alcohol intake characterized by a negative social, occupational, or health impact. The Diagnostic and Statistical Manual of Mental Disorders IV described two distinct disorders: Alcohol abuse and alcohol dependence, while the fifth edition merges the two disorders, into a single disorder called AUD, or AUD, with subclasses ranging from mild to severe. However, binge drinking is considered a pattern based on heavy episodic alcohol intake (equal to $0.08 \mathrm{~g} / \mathrm{dl}$ or higher) in a brief period of time (14).

Adjoined diagnoses include AWS, evolving from Early Withdrawal Syndrome, with a risk of Withdrawal fits, leading to Delirium Tremens and with a chance of Protracted Withdrawal Syndrome. Delirium Tremens is the most severe and life-threatening outcome of AWS. The major risks thereof are malignant arrhythmia, respiratory arrest, severe electrolyte imbalance, prolonged seizures and trauma (15).

Alcohol liver disease (ALD) encompasses a range of disorders including steatosis, hepatitis, fibrosis, liver cirrhosis and hepatocellular carcinoma. Furthermore, liver failure can lead to hepatic encephalopathy (HE), a neuropsychiatric disorder characterized by an altered state of consciousness due to a buildup of toxins (ammonia) (14). When suspecting liver disease, a liver panel is of great importance: aspartate aminotransferase (AST), alanine aminotransferase (ALT), alkaline phosphatase (AP) and bilirubin.

The absorption of alcohol is a rapid process which distributes the alcoholthroughout the body, depending on the water content of the tissues. The two-carbon molecules are only able to interact with other biomolecules via hydrogen bonding and weak hydrophobic interactions, limiting its potency $(14,16)$.

The first step in metabolizing alcohol is oxidation, resulting in acetaldehyde, thenin acetate and finally citric acid. This process involves two enzymes: Alcohol dehydrogenase (ADH) and aldehyde dehydrogenase (ALDH). Acetaldehyde has been linked to behavioral and physiological effects previously attributed to alcohol; when administered to laboratory animals it leads to incoordination, memory impairment, and sleepiness $(17,18)$. A third enzyme, Acetyl-CoA contributes to the citric acid cycle producing cellular energy in the form of ATP, ADP, Pi; releasing water and carbon dioxide as byproducts. Alcohol metabolism occurs differently between individuals because there is a genetic variation in coding the enzymes. The mentioned differences mean greater risk for alcohol-related pathology, whereas other individuals may be at least somewhat protected from the harmful effects of ethanol. For example, individuals with gene varieties for fast ADH or slow ALDH, which retards production of acetaldehyde, have the tendency to drink less $(16,18)$.

The liver has the highest concentration of enzymes, making it the ideal site for the metabolism of ethanol (19). Ethanol oxidative metabolism via intracellular signaling pathways has a negative impact on the transcriptional control of several genes, resulting in fat accumulation, fibrogenesis and activation of innate and adaptive immunity. Alcohol abuse inhibits natural killer cells that are cytotoxic leading to carcinogenic cells $(20,21)$. Therefore, chronic uptake exerts the liver, making it more vulnerable to disease, while binge drinking is a risk factor for advanced liver disease $(14,22)$.

The increase of energy due to the citric acid cycle stimulates dopaminergic neural activity. The resulting dopamine signals the reward pathway which translates into a sense of wellbeing. On the contrary glutamatergic neural activity is suppressed leading to anxiety and unease. These positive 
responses to alcohol use are significant to social alcohol consumption (23). Therefore, the actions of ethanol in the central nervous system explain behavioral changes, such as stimulant followed by depressant effects as well as chronic changes in behavior, including tolerance, compulsive drinking, and addiction (17). Furthermore, substance abuse involves a series of voluntary acts associated with enduring changes in brain function related to automatized behavior. Apart from the variation in metabolic enzymes, it is believed that genes influence other aspects of addiction such as learning or behavioral disinhibition/impulsivity $(22,24)$.

Idiosyncratic alcohol intoxication represents the development of manifestations following the ingestion of small amounts of alcohol (usual). Psychotic clinical manifestations appear including confusion, illusions, delusional ideation, and visual hallucinations. Other manifestations may include psychomotor agitation, aggressive, impulsive behavior, or suicidal ideation. These manifestations last a short period of time and are followed by a prolonged period of sleep and amnesia on waking. The causes are unknown, but it is more common in those with high levels of anxiety (25).

Hypotheses regarding consumption have been previously made: Alcohol produces sufficient disorganization and loss of control, releasing aggressive impulses; brain damage (especially postencephalic or post-traumatic) predisposes to alcohol intolerance, which can lead to abnormal behavior after ingesting small amounts of alcohol.

Idiosyncratic alcohol intoxication has the following precipitating factors: Advanced age, overwork, concomitant use of sedative-hypnotics. Treatments should include protection of the patient and persons related to the individual; restraint, which may be necessary, but difficult to achieve due to sudden onset; and/or Haloperidol i.m. for aggression control. Differential diagnosis to be considered includes complex partial epilepsy.

Many people with idiosyncratic alcohol intoxication have temporary discharges on the EEG after ingesting small amounts of alcohol.

The underlying pathophysiology of AWS is hyperexcitation within the central nervous system (26). If chronic alcohol use is abruptly discontinued, the metabolism fails and is no longer capable of responding rapidly enough to compensate. Chronic alcohol consumption leads to the development of physical tolerance and dependence, which may result from compensatory functional changes through downregulation of GABA receptors and increased expression of NMDA receptors with more glutamate production to maintain the central nervous system (CNS) transmitter homeostasis. A sudden decrease of alcohol consumption exposes these changes resulting in a glutamate-mediated excitation in the central nervous system, leading to autonomic hyperactivity and neuropsychiatric complications, such as delirium and seizure (generalized tonic-clonic). Seizures are largely mediated in the brainstem by abrogating the tonic inhibitory effect of the GABAergic delta subunits. During withdrawal, a rise in dopamine levels contributes to the clinical manifestations of hyperarousal and hallucinations $(27,28)$.

Wernicke's syndrome (alcoholic encephalopathy) is an acute neurological disorder characterized by ataxia, vestibular dysfunction, ophthalmoplegia, nystagmus, anisocoria (usually ocular signs are bilateral), and disturbances of consciousness such as a degree of confusion, or a degree of temporary disorientation. It can resolve spontaneously in a few days or weeks or progress to Korsakoff syndrome. Injuries to the mammillary bodies occur in Korsakoff syndrome. Causes of Korsakoff syndrome include chronic alcoholism (vitamin B1 deficiency), acute intoxication (CO), tumors of the mammillary and hypothalamic region, craniocerebral trauma, senile dementia, gastric cancer, or severe dietary deficiency. In these cases, judgment and reasoning remain intact. Such cases are manifested by fixation amnesia (anterograde), confabulations and false admissions, some indifference, amusement (because he is not aware of amnesia), accentuated polyneuritis in the lower limbs, variable, usually with flaccid paralysis, abolition of ROT, sensory disorders, pain on the nerve pathways, and trophic disorders. The Wernicke-Korsakoff syndrome may have a slow onset, with apathy, depression, dysmnesia, headache and other pseudoneurotic signs. The usual treatment consists of Thiamine p.o. $100 \mathrm{mg} \mathrm{2-3} \mathrm{times/day} \mathrm{for}$ 3-12 months. Very few patients recover completely. A substantial proportion experience improvement in cognitive abilities (with Thiamine and nutritional support). Many have a chronic course of dementia (29).

Guidelines in treating AWS recommend providing medications with GABA receptor activity. Thus, first-line treatment consists of Benzodiazepines (BZD) with rapid escalation of dosing. Benzodiazepines modulate the binding of GABA to the GABA-A receptor, favoring an inhibitory effect similar to ethanol (27). The metabolism of all BZD occurs in the liver through oxidation and in some cases through glucuronidation. Based on their active metabolites BZDs may be categorized according to the duration of their effect: Short acting $(<10 \mathrm{~h})$ : Lorazepam, Oxazepam and Midazolam; intermediate acting (10-24 h): Clonazepam; long acting (>24 h): Clorazepate and Diazepam (27).

BZD loading is a strategy used to favor the pharmacokinetics of this drug to achieve a rapid effect followed by sustained benefit over a period of days (30). Doses in the elderly should be $50 \%$ less than younger adults.

Parenteral thiamine, antiepileptic drugs (Carbamazepine or Phenobarbital) and in some cases neuroleptics (Haloperidol) may be required based on severity and the presence of complications including delirium, seizures and convulsion. Other medications such as $\beta$-antagonists may offer additional benefit in selected patients but should not be used as a monotherapy. Supportive treatment is not to be neglected $(8,31)$.

Transferring the patient to an intensive care unit should be considered when resistant forms of AWS are encountered. In these instances, Propofol can be used as an agent to decrease the effects of withdrawal $(26,28)$. Dexmedetomidine is a well-tolerated efficient alternative to classic withdrawal treatment in the case of refractory symptoms $(26,31)$.

Patients with ALD are likely to be further compromised by the potential hepatocytotoxicity of some pharmacological agents. Subsequently, liver disease comes with a loss in function which decelerates the breakdown of medication. Both hepatic blood flow and liver enzyme activity can be affected by liver disease. That being the case, not only pharmacokinetics, but also pharmacodynamics are perturbed. In said cases the treatment of neurological disorders is prioritized with the fine balance of not compromising liver function $(32,33)$. 
The relationship between alcohol consumption, seizures and epilepsy is still insufficiently explored. Most studies indicate that between 5 and $10 \%$ of alcoholics develop seizures throughout their lives. Higher rates can be observed among alcohol-dependent patients, but with an excessively high level of alcohol consumption for relatively longer periods, i.e., decades. Epileptic seizures that are not caused by alcohol, but represent a comorbidity of epilepsy with alcoholism must be differentiated from acute, symptomatic seizures, which occur in relation to alcohol consumption. Seizures associated with withdrawal syndrome usually occur within $48 \mathrm{~h}$ of last consumption (34).

The same exposure that, in its extreme form, leads to a symptomatic acute seizure, can lead to small doses for seizures in individuals already known to have epilepsy. Thus, in clinical practice, the boundary between provoked and unprovoked seizures may be blurred.

\section{Medication and protocols}

No evidence-based guidelines or protocols exist for prescribing medication in patients suffering from ALD (35). Protein binding, metabolism, bioavailability, extraction ratios, excretion route, and half-life must be taken into consideration as well as frequently repeating liver panels.

Acute alcohol consumption does not trigger seizures, while chronic consumption leads to the development of tolerance and physical dependence; thus, compensatory functional changes lead to the downregulation of GABA receptors (36) and greater expression of NMDA receptors (37). Abrupt discontinuation of alcohol can expose these changes, rapidly causing excess glutamate and a decrease in the tonic inhibitory effect of delta aminolevulinic acid, which is a potent gabaergic agonist. The resulting neuronal overactivity can lead to neuropsychiatric and autonomic nervous system seizures and complications in the form of delirium tremens (34).

Kindling represents the phenomenon by which a weak chemical or electrical stimulus, which would not normally cause a significant behavioral response, administered repeatedly, triggers such a reaction. Kindling results from alterations in neurotransmitters (most often GABA and NMDA) which, in turn, increase cerebral excitability, favoring new convulsive episodes (38).

The kindling phenomenon indicates that $4 \%$ of CT scans of patients with seizures secondary to alcohol withdrawal show structural lesions. Recurrence of seizures at 6 months is $41 \%$ and this increases over time to $51 \%$ in 3 years. New episodes of withdrawal can cause secondary damage to cerebral excitability (kindling), damage that seems to be cumulative.

Although the percentage of epileptics in developed countries is $0.7 \%$, and the risk of a lifelong epileptic seizure is $2-3 \%$, it is estimated that $15 \%$ of alcoholics are bound at some point to experience a seizure (39).

Benzodiazepine. It is common knowledge that benzodiazepines are safer than earlier sedatives used for AWS, such as barbiturates, chloral hydrate and paraldehyde, yet they remain controversial when used in liver disease (40).

Several studies suggest that the pharmacodynamics of some drugs may be altered in cirrhotic patients resulting in unusual adverse effects that require dosage adjustment as well as prolonged half-times. Such drugs include opiates, benzodiazepines, NSAIDs and diuretics (41). Benzodiazepines can also be used in decompensated cirrhosis with caution (42).

According to the available data, cognitive complications occur more frequently in elderly patients suffering from withdrawal making the treatment of AWS more challenging. A common approach in treating elderly patients or those with significant ALD who may have an increased accumulation and decreased clearance of the long-acting benzodiazepines is using Lorazepam or Oxazepam instead (31). Lorazepam is a short-acting benzodiazepine and has no active metabolites favoring its use in ALS. However short-acting benzodiazepines may be less effective in preventing seizures and more prone to produce discontinuation symptoms if not dosed accordingly (43). Other studies have found that Lorazepam is non-inferior to Chlordiazepoxide in reducing alcohol withdrawal symptoms (44).

A study conducted on patients diagnosed with liver cirrhosis who had treatment with benzodiazepines between 3 and 10 days previously had a severe risk of developing first-time hepatic encephalopathy (HE). Cirrhosis patients who had been using benzodiazepines for just one or two days or continued use for more than 28 days did not have such an excess risk (45). A previous article suggested that although it was believed that benzodiazepine-associated GABA-A receptors were unaltered in humans and rats with HE, 'endogenous benzodiazepines' seem to have no involvement in the pathogenesis of hepatic encephalopathy. By contrast non-benzodiazepine GABA-A receptor complex modulators (e.g., neurosteroids), were found in brain in human and experimental HE (46).

Antiepileptic drugs. Anticonvulsants can be used in patients with decompensated liver disease with caution, although newer generation antiepileptic agents should be first-line. New generation antiepileptic drugs (Levetiracetam, Lacosamide, Topiramate, Gabapentin and Pregabalin) either do not have or require a minimal hepatic metabolism making them prime contenders. Hepatotoxicity is a rare and unexpected side effect of modern antiepileptic drugs as well as for Phenobarbital and Ethosuximide. However, pharmaceuticals that require high hepatic metabolism associated with well-recognized liver toxicity such as Valproic acid, Phenytoin and Felbamate, should be used as a final resort. Intravenous Levetiracetam and Lacosamide are a worthy second-line therapy after benzodiazepines fail to control seizures in status epilepticus $(42,47)$.

According to a study by Lynn et al (48), between 2004 and 2013, 225 (2\%) of registered cases had a documented history of epilepsy, of which 82 (36\%) recorded epilepsy as the cause of death. Of these 82 deaths, the majority were male (60-73\%) and for half of them the median age was 47 years or younger. A post-mortem toxicology report was available for $65(79 \%)$ of these deaths, of which over two-thirds (44-68\%) did not have antiepileptic drugs present in the toxicological findings. More than two-thirds of these deaths (31-70\%) were among cases known to be addicted to alcohol. The high percentage of individuals with a diagnosis of alcohol dependence who died as a result of epilepsy and who did not have antiepileptic drugs in the treatment scheme at the time of death, highlights the need for preventive measures for this at-risk group. 
Epileptic seizures usually occur in individuals who have abused alcohol for a period of $\geq 10$ years; thus, long-term change in brain function or structure is inevitable.

Other types. Propofol is favored to benzodiazepines or opioids in the case of decompensated cirrhosis due to its short half-life as well as its lower risk of inducing encephalopathy. Even so Fentanyl elimination is near normal in cirrhosis and can also be used for sedation (42).

Regarding adjuvant treatment, antiemetic agents (Metoclopramide) require significant dose reduction in patients suffering with cirrhosis. Proton pump inhibitors (halved) are preferred over $\mathrm{H} 2$ receptor blockers (9).

\section{Correlation of treatment under psychiatric environment}

One of the most exemplifying cases is of a patient, known to psychiatric services for about three years, entering the psychiatric circuit in November 2017 (at the age of 42). Symptoms identified at the clinic included irritability, tremor of the extremities, temporary disorientation spatial, symptomatology developed against the background of chronic ethanol consumption.

At the time of the patient's entry into the psychiatric circuit, the diagnoses were: Organic affective disorder and mental and behavioral disorders due to alcohol use; addiction syndrome; toxic liver disease with disfunctions at this level, Vitamin B deficiency. The initial treatment administered was for easier withdrawal of weaning: Carbamazepine for lowering craving, hepatic trophic group Silymarin, an adjuvant in the therapy of acute and chronic liver diseases (Metaspar), essential phospholipids and vitamin complex, to prevent demyelination polyneuropathy, as well as Wernicke-Korsakoff syndrome, the patient being informed about the need to receive daily $150-200 \mathrm{mg}$ of vitamin B1 for 1-2 years.

The last hospitalization occurred in the middle of covid-19, after midnight, around 1.00 a.m., at the Emergency Room; the last diagnosis of organic personality disorder, being completed with that of ethanol withdrawal syndrome. At admission, the following information was noted on the observation sheet: Uncomplicated withdrawal, with tremor in the extremities, psychomotor anxiety and hypnotic disorders.

The patient's personal physiological and pathological history should also be taken into account: Gastritis, as well as pancreatitis, following chronic consumption of ethanol in the last days before hospitalization. The patient was treated with antispasmodic and nonsteroidal anti-inflammatory drugs as needed, due to diffuse abdominal pain. In the context of the covid-19 pandemic, the patient could not address any gastroenterology service. Hypertension was also present in treatment with $10 \mathrm{mg}$ of angiotensin converting enzyme inhibitor.

Among the collateral hereditary antecedents, we noted: Father with mixed type of dementia (Alzheimer's and vascular, multiple strokes in the antecedents) and mother who died at the age of approximately 30 (rapid stroke).

From the point of view of living and working conditions, the patient did not work and was not medically insured. He was on disability commission but did not receive it. Consequently, the medical insecurity of patients consuming chronic ethanol, which over time develops important somatic complications, is a focal point of tertiary prevention.

From the objective examination of the general condition at hospitalization of the patient, we noted: The state of preserved consciousness, jaundice teguments, mucosa and sclera, oxygen saturation $95 \%$, BP 145/95 $\mathrm{mmHg}$, ventricular rate $100 \mathrm{bpm}$; at the level of the digestive system: abdomen relaxed by the adipose pedicle, mobile with breathing, painless spontaneously and on palpation, without signs of acute abdomen. The patient agreed to collect Ag HBs and Ac HVC tests, which were negative. The psychiatric approach involved administration of Diazepam $30 \mathrm{mg}$ i.m, intravenous infusion of Arginine $1,000 \mathrm{ml}$ and vitamin therapy (200 mg B1 and $200 \mathrm{mg} \mathrm{B6}$ ), Trazodone $150 \mathrm{mg}$ as a sleep inducer, hepatic drugs such as Silymarin and Metaspar, with maintenance of Enalapril at a dose of $20 \mathrm{mg}$.

The patient's somatic condition progressively deteriorated, and on May 17, around noon, at the emergency room the following was noted: Marked jaundice, severe pain on palpation in the right hypochondrium, oxygen saturation of $89 \%$, fever $38.5^{\circ} \mathrm{C}, \mathrm{BP}=125 / 80 \mathrm{mmHg}, \mathrm{AV}=130 \mathrm{bpm}$. The patient was conscious, cooperative, coherent, spatially temporally oriented, self- and allo-psychic, with obesity predominantly at the abdomen level. Three soft consistency stools were discharged during the night. The patient was isolated and assigned dedicated personnel, an intramuscular nonsteroidal anti-inflammatory drug was administered and it was decided to collect samples for SARS-COV-2 testing, according to the protocol established. The next day, the Covid-19 test was negative.

The patient was evaluated by the attending physician, who supplemented the usual (repeated) tests with the collection of: ESR, PCR, Quick time, prothrombin time (PT), INR, Fibrinogen, direct and total bilirubin. Surgical examination, with abdominal ultrasound scanner, diagnosis of toxic hepatitis was superimposed on liver cirrhosis, with hepatosplenomegaly (left lobe diameter $10.2 \mathrm{~cm}$, right lobe diameter $19.2 \mathrm{~cm}$, antero-posterior spleen diameter $14 \mathrm{~cm}$ ).

The internal medical examination supported the same diagnosis, i.e., mild macrocytic anemia, with hemoglobin $8.5 \mathrm{mg} / \mathrm{dl}$, leukocytosis. The patient was afebrile with hepatosplenomegaly, liver and spleen were sensitive to palpation, and discrete leg edema and fine flapping tremor were noted.

Given the situation of the SARS-COV-2 pandemic, multiple gastroenterology clinics did not have places available to take the patient. Interdisciplinary investigation was required in one of the gastroenterology clinics, a consultation that confirmed that the patient is a chronic consumer of alcohol, was cholecystectomized, with altered liver function at the time of consultation. The patient was conscious, spatially temporally oriented, with no signs suggestive of hepatic encephalopathy, with jaundiced skin and mucous membranes, palpable abdomen through the adipose pedicle, mobile with respiration, sensitive to palpation in the right hypochondrium, without signs of peritoneal irritation. Leukocytosis, hypoalbuminemia, cytolysis and hepatic cholestasis, mild macrocytic hyperchromic anemia were identified. Abdominal ultrasound indicated: Hyperechoic liver, with irregular contour, homogeneous spleen, normal biliary-pancreatic tract, normal portal vein, without free fluid in the peritoneal cavity. The diagnosis was acute alcoholic 
hepatitis superimposed on a toxic nutritional liver cirrhosis, Child-Pugh class B (7-8 points), MELD 20 (model of end-stage liver disease), Maddrey score 36, and FD >32 (indication for corticotherapy). Hepatocellular jaundice was confirmed and the following recommended: Parenteral hydration with Ringer $1,000 \mathrm{mg} / \mathrm{ml}$, Aminoven $50 \mathrm{ml}$, Albumin $50 \mathrm{ml}$ or 2/day with Hydrocortisone hemi-succinate $100 \mathrm{mg}$ or $2 /$ day, Potassium chloride $20 \mathrm{ml}$ or 2/day, hypo-protein regimen, hypolipidemic, hyposodate with the administration of 2 liters of fluids daily. In addition, performing dynamic tests, as well as digestive endoscopy after resolving acute toxic hepatitis was recommended. The patient received treatment with Albumin $100 \mathrm{ml} / \mathrm{day}$, doubled by Hydrocortisone hemi-succinate $200 \mathrm{ml} /$ day for seven days, remaining in the psychiatric service given the situation of the pandemic with SARS-COV-2 and the objective temporary impossibility to be taken in by a gastroenterology service.

Among the dynamic analyzes of the patient, we noted: GGT $=2173 \mathrm{U} / 1$, with hyponatremia $=131 \mathrm{mmol} / 1, \mathrm{GOT}=256 \mathrm{U} / \mathrm{l}$, $\mathrm{GPT}=137 \mathrm{U} / \mathrm{l}$ and that total cholesterol $=228 \mathrm{mg} / \mathrm{dl}$ and triglycerides $=395 \mathrm{mg} / \mathrm{dl}$. Blood smear by cytological examination revealed: Anisocytosis with the presence of macrocytes, anisochromia, rare target erythrocytes, rare spherocytes, erythroblasts $=8 \%$, rare macrothrombocytes. HBsAg and $\mathrm{HCV}$ Ac performed on May 15 were negative, the percentage of monocytes on HLG was slightly increased to $10.9 \%$, with the increase of the percentage of immature granulocytes to $1.23 \%$, with the decrease of erythrocytes to $2.78^{*} 10^{6} / \mathrm{microl}$. Hemoglobin was $9.9 \mathrm{~g} / \mathrm{dl}$, with a Hematocrit value of $28.5 \%$, with an increase in mean erythrocyte volume at $102.5 \mathrm{fl}$ and mean erythrocyte hemoglobin at $35.6 \mathrm{pg}$. In addition, the average platelet volume was $12.1 \mathrm{fl}$.

Dynamic tests performed after three days, indicated a decrease in albumin to $2.61 \mathrm{~g} / \mathrm{dl}$, with direct bilirubin reaching $13.3 \mathrm{mg} / \mathrm{dl}$, of which total bilirubin reached $16.4 \mathrm{mg} / \mathrm{dl}$. The value of Chlorine on the ionogram was $93 \mathrm{mmol} / \mathrm{l}$, creatine kinase was within normal limits, as was alkaline phosphatase, and the c3 and c4 fractions of the complement (harvested as markers of inflammation). The phosphorus value was reduced to $2.17 \mathrm{mg} / \mathrm{dl}$. The high value of the GGT was maintained=1,386 U/l. The immunogram revealed immunoglobulin $\operatorname{IgA}=5.19 \mathrm{~g} / 1$, lactic dehydrogenase $=470 \mathrm{U} / 1$, total lipids $=825 \mathrm{mg} / \mathrm{dl}, \mathrm{C}$-reactive protein increased to $201.6 \mathrm{mg} / \mathrm{l}$, decrease of total proteins to $6 \mathrm{~g} / \mathrm{dl}$, potassium and magnesium values remained low, as did the value of sodium. TGO was normalized, leaving TGP at $209 \mathrm{U} / \mathrm{l}$. Coagulation samples were modified: APTT=35.7 sec, Fibrinogen $=430 \mathrm{mg} / \mathrm{dl}$, prothrombin time $=13.5 \mathrm{sec}$ (prothrombin activity within normal limits), INR within normal limits. Leukocytosis was maintained at $18,890^{*} 10^{3} / \mathrm{microl}$, with $11.480^{*} 10^{3} / \mathrm{microl}$ neutrophils, remaining the percentage of immature granulocytes increased by $8.5 \%$, with the decrease of erythrocytes at $2.39^{*} 10^{6} / \mathrm{microl}$, Hemoglobin decreasing $=8.5 \mathrm{~g} / \mathrm{dl}$, Hematocrit $=24.7 \%$, mean erythrocyte volume $=103 \mathrm{fl}$, mean erythrocyte hemoglobin=35.6 pg, ESR=84 mm/h.

The dynamic repetition of the analyzes after two more days, supports the continuation of the hepatic cytolysis process, with the total Bilirubin reaching $23.7 \mathrm{mg} / \mathrm{dl}$, of which direct Bilirubin=18.7 mg/dl, intense jaundice serum, with decreased magnesium and potassium. On May 22, leukocytosis decreased to $11,180^{*} 10^{3} / \mathrm{microl}$, with low maintenance of erythrocytes at $2.36^{*} 10^{6} / \mathrm{microl}$, Hemoglobin at $8.7 \mathrm{~g} / \mathrm{dl}$, Hematocrit at $25.6 \%$, volume average erythrocyte increased to $108.5 \mathrm{fl}$, with average erythrocyte hemoglobin=36.9 pg, Albumin=2.81 g/dl. GGT1184 U/l, TGO=252 U/1, TGP=137 U/l. The prothrombin time was increased to $13.8 \mathrm{sec}$.

After 11 days, Albumin remainedlow, but was increasing compared to previous values, reaching $2.87 \mathrm{~g} / \mathrm{dl}$, total bilirubin at $3.54 \mathrm{mg} / \mathrm{dl}$ and direct bilirubin was $2.47 \mathrm{mg} / \mathrm{dl}$, with a decrease in total calcium as well as ionic calcium. GGT reached $358 \mathrm{U} / 1, \mathrm{TGO}=99 \mathrm{U} / 1, \mathrm{TGP}=109 \mathrm{U} / 1$, prothrombin time $=13.2 \mathrm{sec}$, prothrombin activity and INR within normal limits, Hemoglobin increased to $9.4 \mathrm{~g} / \mathrm{dl}$, maintaining a low erythrocyte count $2.51^{*} 10^{6} / \mathrm{microl}$.

It should be mentioned that such cases can be the subject of hospitalization in the psychiatry service, the patient's pathology starting with a withdrawal syndrome, which was not complicated in terms of altered consciousness (the patient not showing delirium tremens phenomenology), instead was complicated in terms of liver pathology, which became acute, by increased bilirubin (both total and direct) to values of $14 \mathrm{mg} / \mathrm{dl}$, respectively, $16-18 \mathrm{mg} / \mathrm{dl}$, with increased GGT to $3,000 \mathrm{mg} / \mathrm{dl}$ and increased transaminases, hepatic cytolysis and inflammation with leukocytosis. From a clinical point of view, the general somatic condition of the patient has progressively altered, presenting lipothymia, as well as fever in the context of hepatic cytolysis. We are talking about an evolution of acute fulminant toxic hepatitis, which required the administration of Albumin, requiring interdisciplinary consultations and collaboration between clinical specialties, to stop the evolution to an alcoholic encephalopathy.

\section{Conclusion}

The interaction of alcohol with medicines, anesthetics, and cross tolerance were examined in this review. Alcoholics need higher doses of anesthetics as they develop greater resistance to anesthetics including antianginal ones, antihypertensive ones, where they potentiate each other; anticoagulants: Alcohol increases the effect of anticoagulants, favoring the appearance of hemorrhages; antibiotics: Many antibiotics cause disulfiramic reactions; neuroleptics: Studies say that neuroleptics are toxic in combination with alcohol; antidepressants: Alcohol impedes their metabolism, causing their accumulation and thus an overdose; psychostimulating substances: Sometimes the reaction is antagonistic, other times it is cumulative, the effect being accentuated; sedatives and hypnotics: Non-drinking alcoholics have an unusual tolerance for these drugs, due to accelerated metabolism. When an individual is intoxicated these drugs accumulate in potentially toxic amounts due to competition with alcohol for the same detoxification mechanisms.

Patients with ALD are likely to be further compromised by the potential hepatocytotoxicity of some pharmacological agents. On that account, having an integrated perspective of the medical case while taking into consideration the underlying illness as well as possible drug interaction, protein binding, metabolism, bioavailability, extraction ratios, excretion route, and half-life is crucial in treating AUD or AWS in a psychiatric institution. The treatment of neurological disorders is prioritized with the fine balance of not compromising 
remainder liver function. A collaborative multidisciplinary team with attentive monitoring is in the patient's best interest.

\section{Acknowledgements}

Not applicable.

\section{Funding}

Not applicable.

\section{Availability of data and materials}

Not applicable.

\section{Authors' contributions}

AT has designed, drafted and revised the review. ST gathered the medical information and gave a final view and structure of the article. AP studied the present area of research and gathered the important information. RVC finalized analysis of the results. All authors read and approved the final manuscript.

\section{Ethics approval and consent to participate}

Not applicable.

\section{Patient consent for publication}

Not applicable.

\section{Competing interests}

The authors declare that they have no competing interests.

\section{References}

1. Becker HC and Mulholland PJ: Neurochemical mechanisms of alcohol withdrawal. Handb Clin Neurol 125: 133-156, 2014.

2. Mowbray O, Quinn A and Cranford JA: Social networks and alcohol use disorders: Findings from a nationally representative sample. Am J Drug Alcohol Abuse 40: 181-186, 2014.

3. Newton-Howes G and Foulds J: Personality disorder and alcohol use disorder: An overview. Psychopathology 51: 130-136, 2018.

4. Gilpin NW and Weiner JL: Neurobiology of comorbid post-traumatic stress disorder and alcohol-use disorder. Genes Brain Behav 16: 15-43, 2017.

5. Boulze-Launay I and Rigaud A: Alcohol and loneliness: In my solitude. Int Forum Psychoanal 29: 65-73, 2020.

6. Brown ES, Carmody TJ, Schmitz JM, Caetano R, Adinoff B, Swann AC and Rush AJ: A randomized, double-blind, placebo-controlled pilot study of naltrexone in outpatients with bipolar disorder and alcohol dependence. Alcohol Clin Exp Res 33: 1863-1869, 2009.

7. Tawa EA, Hall SD and Lohoff FW: Overview of the genetics of alcohol use disorder. Alcohol Alcohol 51: 507-514, 2016.

8. Hasin DS, Stinson FS, Ogburn E and Grant BF: Prevalence, correlates, disability, and comorbidity of DSM-IV alcohol abuse and dependence in the United States: Results from the national epidemiologic survey on alcohol and related conditions. Arch Gen Psychiatry 64: 830-842, 2007.

9. Yoon YH and Chen CM: Surveillance report \#105: Liver cirrhosis mortality in the United States: National, state, and regional trends, 2000-2013. Bethesda, MD, National Institute on Alcohol Abuse and Alcoholism (NIAAA), 2016. Available from: http://pubs.niaaa.nih.gov/publications/Surveillance105/Cirr13.pdf.

10. Schuckit MA: Recognition and management of withdrawal delirium (delirium tremens). N Engl J Med 371: 2109-2113, 2014.
11. Muncie HL Jr, Yasinian Y and Oge' L: Outpatient management of alcohol withdrawal syndrome. Am Fam Physician 88: 589-595, 2013.

12. World Health Organization: Status report on alcohol consumption, harm and policy responses in 30 European countries. Copenhaga, WHO, 2019.

13. IovuMB and Breaz MA: The prevalence and burden of mental and substance use disorders in romania: findings from the global burden of disease study 2016. Psychiatr Danub 31: 457-464, 2019.

14. Ventura-Cots M, Watts AE and Bataller R: Binge drinking as a risk factor for advanced alcoholic liver disease. Liver Int 37: 1281-1283, 2017.

15. Trifu S, Marica S, Braileanu D, Carp EG and Gutt AM: Teaching psychiatric concepts of neurosis, psychosis and borderline pathology. Conceptual boundaries. Proc Soc Behav Sci 203: 125-129, 2015.

16. Cederbaum AI: Alcohol metabolism. Clin Liver Dis 16: 667-685, 2012.

17. Deitrich R, Zimatkin S and Pronko S: Oxidation of ethanol in the brain and its consequences. Alcohol Res Health 29: 266-273, 2006.

18. The National Institute on Alcohol Abuse and Alcoholism: Alcohol metabolism: An update. Alcohol Res Health 72: 1-5, 2007

19. Wilson DF and Matschinsky FM: Ethanol metabolism: The good, the bad, and the ugly. Med Hypotheses 140: 109638, 2020.

20. Ceni E, Mello T and Galli A: Pathogenesis of alcoholic liver disease: Role of oxidative metabolism. World J Gastroenterol 20: 17756-17772, 2014.

21. Costea R, Zarnescu NO, Vasiliu EC, Minca D, Sajin M and Neagu S: B1 versus B2 Dukes stage rectal cancer: Prospective evaluation of 87 patients in a single institution and quest for re-evaluation of the current proto. Hepatogastroenterology 59: 98-100, 2012

22. Volkow ND, Koob G and Baler R: Biomarkers in substance use disorders. ACS Chem Neurosci 6: 522-525, 2015.

23. Trifu S, Carp EG and Nadoleanu A: Alcohol as a substitute, mask of depression and 'antidote' of narcissism. Eur Proc Soc Behav Sci 31: 986-994, 2017

24. Cabral Barata P, Oliveira CFP, Lima de Castro S and Rocha da Mota AMP: A systematic review on substance addiction: Medical diagnosis or morality flaw? Eur J Psychiatry 33: 143-151, 2019.

25. Jung YC and Namkoong K: Alcohol: Intoxication and poisoningdiagnosis and treatment. Handb Clin Neurol 125: 115-121, 2014.

26. Long D, Long B and Koyfman A: The emergency medicine management of severe alcohol withdrawal. Am J Emerg Med 35: 1005-1011, 2017.

27. Jesse S, Bråthen G, Ferrara M, Keindl M, Ben-Menachem E, Tanasescu R, Brodtkorb E, Hillbom M, Leone MA and Ludolph AC: Alcohol withdrawal syndrome: Mechanisms, manifestations, and management. Acta Neurol Scand 135: 4-16, 2017.

28. Kattimani S and Bharadwaj B: Clinical management of alcohol withdrawal: A systematic review. Ind Psychiatry J 22: 100-108, 2013.

29. Isenberg-Grzeda E, Kutner HE and Nicolson SE: WernickeKorsakoff-syndrome: Under-recognized and under-treated. Psychosomatics 53: 507-516, 2012.

30. Muzyk AJ, Leung JG, Nelson S, Embury ER and Jones SR: The role of diazepam loading for the treatment of alcohol withdrawal syndrome in hospitalized patients. Am J Addict 22: 113-118, 2013.

31. Perry EC: Inpatient management of acute alcohol withdrawal syndrome. CNS Drugs 28: 401-410, 2014.

32. Bautista AP: Liver injury during alcohol use and withdrawal. In: Comprehensive Handbook of Alcohol Related Pathology. Preedy VR and Watson RR (eds). Academic Press, Cambridge, pp491-500, 2005.

33. Rodighiero V: Effects of liver disease on pharmacokinetics. An update. Clin Pharmacokinet 37: 399-431, 1999.

34. Samokhvalov AV, Irving H, Mohapatra S and Rehm J: Alcohol consumption, unprovoked seizures, and epilepsy: A systematic review and meta-analysis. Epilepsia 51: 1177-1184, 2010.

35. Trifu S and Trifu AD: Receptor profiles of atypical antipsychotic molecules. UPB Sci Bull 82: 113-128, 2020.

36. Frye GD and Fincher AS: Effect of ethanol on gamma-vinyl GABA-induced GABA accumulation in the substantia nigra and on synaptosomal GABA content in six rat brain regions. Brain Res 449: 71-79, 1988.

37. Tsai G and Coyle JT: The role of glutamatergic neurotransmission in the pathophysiology of alcoholism. Annu Rev Med 49: 173-184, 1998. 
38. Pinel JP and Van Oot PH: Generality of the kindling phenomenon: Some clinical implications. Can J Neurol Sci 2: 467-475, 1975.

39. Wojnar M, Bizoń Z and Wasilewski D: Assessment of the role of kindling in the pathogenesis of alcohol withdrawal seizures and delirium tremens. Alcohol Clin Exp Res 23: 204-208, 1999.

40. Shaw GK: Detoxification: The use of benzodiazepines. Alcohol Alcohol 30: 765-770, 1995

41. Delcò F, Tchambaz L, Schlienger R, Drewe J and Krähenbühl S: Dose adjustment in patients with liver disease. Drug Saf 28: 529-545, 2005.

42. Amarapurkar DN: Prescribing medications in patients with decompensated liver cirrhosis. Int J Hepatol 2011: 519526, 2011.

43. Kraemer KL, Conigliaro J and Saitz R: Managing alcohol withdrawal in the elderly. Drugs Aging 14: 409-425, 1999.

44. Ramanujam R, Padma L, Swaminath G and Thimmaiah RS: A comparative study of the clinical efficacy and safety of lorazepam and chlordiazepoxide in alcohol dependence syndrome. J Clin Diagn Res 9: FC10-FC13, 2015.
45. Grønbæk L, Watson H, Vilstrup H and Jepsen P: Benzodiazepines and risk for hepatic encephalopathy in patients with cirrhosis and ascites. United European Gastroenterol J 6: 407-412, 2018.

46. Ahboucha $S$ and Butterworth RF: Role of endogenous benzodiazepine ligands and their GABA-A-associated receptors in hepatic encephalopathy. Metab Brain Dis 20: 425-437, 2005.

47. Vidaurre J, Gedela S and Yarosz S: Antiepileptic drugs and liver disease. Pediatr Neurol 77: 23-36, 2017.

48. Lynn E, Lyons S, Langan Y, Craig S and Doherty C: The role of alcohol dependency in deaths among people with epilepsy recorded by the national drug-related deaths index (NDRDI) in Ireland, 2004-2013. Seizure 45: 52-55, 2017.

(i) (2) This work is licensed under a Creative Commons Attribution-NonCommercial-NoDerivatives 4.0 International (CC BY-NC-ND 4.0) License. 